PROCEEDINGS OF THE

AMERICAN MATHEMATICAL SOCIETY

Volume 135, Number 3, March 2007, Pages 767-776

S 0002-9939(06)08821-6

Article electronically published on October 2, 2006

\title{
FIXED POINT INDICES AND INVARIANT PERIODIC SETS OF HOLOMORPHIC SYSTEMS
}

\author{
GUANG YUAN ZHANG \\ (Communicated by Carmen C. Chicone)
}

\begin{abstract}
This note presents an intuitive method to study center families of periodic orbits of complex holomorphic differential equations near singularities, based on some iteration properties of fixed point indices. As an application of this method, we will prove Needham's theorem in a more general version.
\end{abstract}

\section{FiXed POINT INDICES OF HOLOMORPHIC MAPPINGS}

Let $\mathbb{C}^{n}$ be the complex vector space of dimension $n$, let $U$ be an open set in $\mathbb{C}^{n}$ and let $f: U \rightarrow \mathbb{C}^{n}$ be a holomorphic mapping. If $p \in U$ is an isolated zero of $f$, say, there exists a bounded open set $V$ with $p \in V \subset \bar{V} \subset U$ such that $p$ is the unique solution of the equation $f(x)=0$ in $\bar{V}$. Then we can define the zero index of $f$ at $p$ by

$$
\pi_{f}(p)=\#\{x \in V ; f(x)=q\},
$$

where $q$ is a regular value of $f$ such that $|q|$ is small enough and \# denotes the cardinality. $\pi_{f}(p)$ is well defined (see [9] or [17] for the details).

If $f: U \rightarrow \mathbb{C}^{n}$ is a holomorphic mapping and $p$ is an isolated fixed point of $f$, then there is a ball $B$ in $U$ centered at $p$ so that $p$ is the unique fixed point of $f$ in $\bar{B}$, in other words, $p$ is the unique zero of the mapping

$$
f-I: \bar{B} \rightarrow \mathbb{C}^{n},
$$

which puts each $x \in \bar{B}$ into $f(x)-x$, and then the fixed point index of $f$ at $p$ is well defined by

$$
\mu_{f}(p)=\pi_{f-I}(p) .
$$

Fixed point indices of holomorphic mappings have the geometric properties stated in the following lemmas (see [16 and [17]). We will denote by $\Delta^{n}$ a ball in $\mathbb{C}^{n}$ centered at the origin.

Lemma 1. Let $f: \Delta^{n} \rightarrow \mathbb{C}^{n}$ be a holomorphic mapping such that $0 \in \Delta^{n}$ is an isolated fixed point of $f$. Then $\mu_{f}(0) \geq 1$, and the equality holds if and only if the Jacobian matrix $f^{\prime}(0)$ of $f$ at 0 has no eigenvalue equal to 1.

Received by the editors May 15, 2005 and, in revised form, October 7, 2005.

2000 Mathematics Subject Classification. Primary 32H50, 32M25, 37C25.

Key words and phrases. Fixed point index, ordinary differential equation, holomorphic differential equation.

The author was supported by Chinese NSFC 10271063 and 10571009.

(C)2006 American Mathematical Society 
Lemma 2. Let $U$ be an open and bounded set in $\mathbb{C}^{n}$ and let $f: \bar{U} \rightarrow \mathbb{C}^{n}$ be a holomorphic mapping such that $f$ has no fixed point on the boundary $\partial U$. Then $f$ has only finitely many fixed points in $U$.

Lemma 3. Let $f: \overline{\Delta^{n}} \rightarrow \mathbb{C}^{n}$ be a holomorphic mapping such that 0 is the unique fixed point of $f$ in $\overline{\Delta^{n}}$. Then for any holomorphic mapping $g: \overline{\Delta^{n}} \rightarrow \mathbb{C}^{n}$ that is sufficiently close to $f$ on the boundary $\partial \Delta^{n}, g$ has only finitely many fixed points in $\Delta^{n}$ and

$$
\mu_{f}(0)=\sum_{\substack{g(x)=x \\ x \in \Delta^{n}}} \mu_{g}(x) .
$$

This lemma gives us an intuitive interpretation of the fixed point index: with the same condition of this lemma, for any holomorphic mapping $g: \overline{\Delta^{n}} \rightarrow \mathbb{C}^{n}$ such that $g$ is sufficiently close to $f$ on the boundary $\partial \Delta^{n}$ and all fixed points of $g$ in $\Delta^{n}$ are simple, $g$ has exactly $\mu_{f}(0)$ distinct fixed points in $\Delta^{n}$. A fixed point $x$ of $g$ is called simple if $\operatorname{det}\left(g^{\prime}(x)-I\right) \neq 0$, say, the Jacobian matrix $g^{\prime}(x)$ has no eigenvalue 1 , where $I$ is the unit matrix.

Let $f: \Delta^{n} \rightarrow \mathbb{C}^{n}$ be a holomorphic mapping such that 0 is a fixed point of $f$. Then for any positive integer $k$, the $k$-th iteration $f^{k}$ of $f$ is well defined in some neighborhood $V_{k}$ of 0 , where $f^{1}=f, f^{2}=f \circ f, f^{3}=f \circ f \circ f$, and so on. The following result can be found in a more general and more precise version in 16 when $n=2$, and when $n>2$ the proof is similar and will be given in the Appendix.

Proposition 1. Let $m>1$ be a prime number and let $f: \Delta^{n} \rightarrow \mathbb{C}^{n}$ be a holomorphic mapping such that $0 \in \Delta^{n}$ is an isolated fixed point of both $f$ and $f^{m}$. If $f^{\prime}(0)$ has an eigenvalue that is a primitive $m$-th root of 1 , then

$$
\mu_{f^{m}}(0)>m .
$$

A complex number $\lambda$ is called a primitive $m$-th root of 1 , if $\lambda^{m}=1$ but $\lambda^{k} \neq 1$ for $k=1, \ldots, m-1$. The following lemma is due to M. Shub and D. Sullivan [14].

Lemma 4. Let $m>1$ be a positive integer and let $\Theta: \Delta^{n} \rightarrow \mathbb{C}^{n}$ be a holomorphic mapping with an isolated fixed point at the origin $0 \in \Delta^{n}$. Assume that for each eigenvalue $\lambda$ of $\Theta^{\prime}(0)$, either $\lambda=1$ or $\lambda^{m} \neq 1$. Then 0 is still an isolated fixed point of $\Theta^{m}$.

In the rest of this section we will consider a holomorphic system

$$
\dot{x}=\frac{d x}{d t}=F(x), x \in \Delta^{n},
$$

where $F: \Delta^{n} \rightarrow \mathbb{C}^{n}$ is a holomorphic mapping such that 0 is an isolated zero of $F$, say, 0 is an isolated singularity of the system. The following result is well known.

Lemma 5. For any $\tau>0$, there is a ball $B \subset \Delta^{n}$ centered at the origin 0 such that the local flow $\phi(t, x)$ of (1.2) is real analytic on $[0, \tau] \times B$, holomorphic with respect to $x, \phi([0, \tau] \times B) \subset \Delta^{n}$ and, putting $\Phi_{\tau}(x)=\phi(\tau, x)$, the Jacobian matrices $\Phi_{\tau}^{\prime}(0)$ and $F^{\prime}(0)$ of $\Phi_{\tau}$ and $F$ at 0 , respectively, are related by

$$
\Phi_{\tau}^{\prime}(0)=e^{\tau F^{\prime}(0)} .
$$

Now, we can prove the following result as a consequence of Proposition 1 . 
Corollary 1. Consider the holomorphic system (1.2) and assume the Jacobian matrix $F^{\prime}(0)$ has an eigenvalue wi, with $\omega \neq 0$ being real. Let $\Phi(x)=\phi\left(\frac{2 \pi}{|\omega|}, x\right)$ be the time $\frac{2 \pi}{|\omega|}$ mapping of the local flow $\phi(t, x)$ of the system. Then 0 is an accumulated fixed point of $\Phi$.

Proof. By Lemma 5, $\Phi$ is well defined and holomorphic in a neighborhood of 0 . To prove the conclusion by contradiction, we assume that 0 is an isolated fixed point of $\Phi$. Then $\mu=\mu_{\Phi}(0)$ is well defined.

Let $m$ be a prime number with $m>\mu_{\Phi}(0)$. Then 0 is an isolated fixed point of both $\Psi(x)=\phi\left(\frac{2 \pi}{m|\omega|}, x\right)$ and the $m$-th iteration $\Psi^{m}$ of $\Psi$, for

$$
\Psi^{m}(x)=\phi\left(\frac{m 2 \pi}{m|\omega|}, x\right)=\Phi(x)
$$

in a neighborhood of 0 . The previous equality also implies that

$$
\mu_{\Psi^{m}}(0)=\mu_{\Phi}(0)=\mu .
$$

On the other hand, it follows from (1.3) that $\Psi^{\prime}(0)$ has an eigenvalue equal to $e^{\frac{2 \pi \omega}{m|\omega|} i}$, which is a primitive $m$-th root of 1 , and then by Proposition 1 .

$$
\mu_{\Psi^{m}}(0)>m>\mu .
$$

This contradicts (1.4). Therefore, 0 cannot be an isolated fixed point of $\Phi$.

This corollary is the key ingredient of our method for studying invariant varieties of holomorphic systems. After some analysis about the fixed point set Fix $(\Phi)$ of $\Phi$ we will be able to prove that $F i x(\Phi)$ contains an analytic variety of complex dimension at least 1 , consisting of 0 and periodic orbits of the same period of the system. Under certain conditions, we will also be able to prove that Fix $(\Phi)$ consists of periodic orbits of the same period $\frac{2 \pi}{|\omega|}$. Here the period always means the least positive period.

\section{Periodically INVARIANT VARIETIES OF HOLOMORPHIC SYSTEMS}

2.1. Centers of planar holomorphic systems. In the history of the qualitative theory of planar ODEs, one of the most interesting problems is to find the condition such that a singularity of an ODE is a center or isochronous center.

Let $U$ be a domain in $\mathbb{R}^{2}$ and let $G \in C^{k}\left(U, \mathbb{R}^{2}\right), k \geq 1$. For the planar ODE

$$
\dot{x}=G(x), x \in U,
$$

an isolated singular point $p \in U$ is called a center if and only if there exists a punctured neighborhood $V \subset U$ of $p$, consisting of periodic orbits of (2.1) surrounding $p$, and $p$ is called an isochronous center if and only if it is a center and all orbits of (2.1) near $p$ have the same period.

Up to now, several classes of systems have been studied extensively, in relation to the existence of isochronous centers (see [2]). Among them, the equation

$$
\dot{z}=P(z), z \in U \subset \mathbb{R}^{2} \cong \mathbb{C},
$$

where $P$ is a complex holomorphic function defined on $U$, were considered in [1], [3], [5], 6], 7], 8], [12], 13] and [15], etc.

The following result about isochronous centers of complex holomorphic equations was given by Gregor [6] in 1958 . 
Theorem 1 (Gregor). Consider the system

$$
\dot{z}=P(z), z \in U \subset \mathbb{C},
$$

where $P$ is a holomorphic function defined on $U$. A simple zero $0 \in U$ of $P$ is a center of system (2.2) if and only if $P^{\prime}(0)$ is pure imaginary. In this case 0 is an isochronous center and the common period of each cycle surrounding 0 is $T=\frac{2 \pi}{\left|P^{\prime}(0)\right|}$.

This result can be found summarized in [7, and several proofs of this theorem can be found in [1], 3], 8], [12] and [15].

2.2. Invariant periodic sets of holomorphic systems in $\mathbb{C}^{n}$. Now, consider an $n$-dimensional complex holomorphic system

$$
\dot{x}=F(x), x \in \Delta^{n},
$$

where $\Delta^{n}$ is a ball centered at the origin 0 in $\mathbb{C}^{n}$ and $F: \Delta^{n} \rightarrow \mathbb{C}^{n}$ is a holomorphic mapping such that 0 is an isolated zero of $F$, say, 0 is an isolated singularity of (2.3).

When $n=2$, as a generalization of Gregor's theorem, Needham and McAllister 11] proved that if

$$
F^{\prime}(0)=\left(\begin{array}{cc}
\mu i & 0 \\
0 & \lambda
\end{array}\right),
$$

where $\mu \neq 0$ is a real number and $\lambda \neq 0$ is a complex number, then there exists a one-dimensional complex manifold consisting of 0 and periodic orbits of (2.3) of the same period.

For arbitrary $n$, Needham [10] proved the following theorem.

Theorem 2 (Needham). If $F^{\prime}(0)$ has a nonzero pure imaginary eigenvalue $\mu i$ and if the other eigenvalues all have nonzero real parts, then there uniquely exists a one-dimensional complex submanifold of a neighborhood of 0 in $\Delta^{n}$, consisting of 0 and periodic orbits of (2.3) of the same period $\frac{2 \pi}{|\mu|}$.

In this note, we shall use Corollary 1 together with some analysis of fixed point sets of certain moment mapping of the local flow of (2.3), to prove Needham's theorem in more general versions.

Theorem 3. There is a complex analytic variety in $\Delta^{n}$ with pure complex dimension at least 1 consisting of 0 and periodic orbits of (2.3) of the same period, if and only if $F^{\prime}(0)$ has a nonzero pure imaginary eigenvalue.

Theorem 4. If $F^{\prime}(0)$ has a nonzero pure imaginary eigenvalue wi and if for any other eigenvalue $\lambda$ of $F^{\prime}(0)$,

$$
\lambda /(\omega i) \neq \pm 2, \pm 3, \ldots,
$$

then there exists a complex analytic variety in $\Delta^{n}$ of pure complex dimension at least 1 , consisting of 0 and periodic orbits of (2.3) of the same period $\frac{2 \pi}{|\omega|}$.

Theorem 5. If $F^{\prime}(0)$ has a nonzero pure imaginary eigenvalue wi and if for any other eigenvalue $\lambda$ of $F^{\prime}(0)$,

$$
\lambda /(\omega i) \neq 0, \pm 1, \pm 2, \ldots,
$$

then there uniquely exists a complex analytic disk $D$ in $\Delta^{n}$ consisting of 0 and periodic orbits of (2.3) of the same period $\frac{2 \pi}{|\omega|}$. 
The term pure dimension means that the variety has the same dimension everywhere, the term analytic disk means that $D$ is a holomorphic embedding of a disk in the complex plane and the uniqueness of $D$ means that any analytic disk satisfying the condition in the theorem coincides with $D$ in a neighborhood of 0 in $\mathbb{C}^{n}$.

Remark 1. The method in this note can also be applied to studying the analyticity of stable manifolds and unstable manifolds of holomorphic differential equations, by parameter transformation of the time $t$ : we can use any line in the complex plane to replace the real line of $t$.

For example, if we replace the time $t$ by $(a+i b) t$ with $b \neq 0$, then the invariant varieties in Theorems 3 to 5 become stable, or unstable, invariant sets in the new systems.

\section{Proof of Theorems 35}

Lemma 6. There exist positive numbers $\delta$ and $T_{0}$, such that for each $T$ in the interval $\left(0, T_{0}\right]$, the system (2.3) has no periodic orbit of period $T$ intersecting $\bar{B}_{\delta}$, where $B_{\delta}=\left\{x \in \mathbb{C}^{n} ;|x|<\delta\right\}$.

Proof. Let $\phi=\phi(t, x)$ be the local flow of (2.3). By Lemma 5 , for any $T_{0}>0$, there is a $\delta>0$, such that $\phi(t, x)$ is real analytic on $\left[0, T_{0}\right] \times \bar{B}_{\delta}$ and complex holomorphic with respect to $x$. We shall show that the conclusion holds for sufficiently small $\delta$ and sufficiently small $T_{0}$.

Otherwise, for any fixed $\delta$ and $T_{0}$, there exist sequences $\left\{\eta_{j}\right\}$ in the interval $(0, \delta)$ and $\left\{t_{j}\right\}$ in the interval $\left(0, T_{0}\right)$ such that

$$
\eta_{j} \rightarrow 0 \text { and } t_{j} \rightarrow 0 \text { as } j \rightarrow \infty,
$$

and that for each $j,(2.3)$ has a periodic orbit $\mathcal{O}_{j}$ of period $t_{j}$ intersecting $B_{\eta_{j}}$.

By (3.1) and the fact that $\phi(0, x) \equiv x$ we have

$$
\max _{x \in \bar{B}_{\delta}, 0 \leq t \leq t_{j}}|\phi(t, x)-x| \rightarrow 0 \text { as } j \rightarrow \infty .
$$

Considering that $\mathcal{O}_{j} \cap B_{\eta_{j}}$ contains infinitely many fixed points of the time $t_{j}$ mapping $\Phi_{t_{j}}(x)=\phi\left(t_{j}, x\right)$ of the local flow $\phi$ and $\eta_{j}<\delta$, we conclude by Lemma 2 that the time $t_{j}$ mapping $\Phi_{t_{j}}$ has a fixed point $x_{j} \in \partial B_{\delta}$ (the boundary of $B_{\delta}$ ), for each $j$. Without loss of generality, assume

$$
\lim _{j \rightarrow \infty} x_{j}=x_{0}
$$

for some $x_{0} \in \partial B_{\delta}$.

We assume that $\delta$ is small enough such that 0 is the unique singularity of (2.3) in $\bar{B}_{\delta}$. Then there exist positive numbers $t_{0}$ and $\theta_{0}$ with $t_{0}<T_{0}$, such that

$$
\left|\phi\left(t_{0}, x_{0}\right)-x_{0}\right|>\theta_{0} .
$$

Thus, by (3.3), for sufficiently large $j$,

$$
\left|\phi\left(t_{0}, x_{j}\right)-x_{j}\right|>\theta_{0} .
$$

On the other hand, since $x_{j}$ is a fixed point of the time $t_{j}$ mapping $\Phi_{t_{j}}$, we have that $\phi\left(t_{j}, x_{j}\right)=x_{j}$ for each $j$, and then, putting $t_{0}=m_{j} t_{j}+r_{j}$ for each $j$, where 
$m_{j}$ is an integer and $0 \leq r_{j}<t_{j}$, we have

$$
\begin{aligned}
& \left|\phi\left(r_{j}, x_{j}\right)-x_{j}\right|=\left|\phi\left(r_{j}, \phi\left(m_{j} t_{j}, x_{j}\right)\right)-x_{j}\right| \\
= & \left|\phi\left(m_{j} t_{j}+r_{j}, x_{j}\right)-x_{j}\right|=\left|\phi\left(t_{0}, x_{j}\right)-x_{j}\right|>\theta_{0} .
\end{aligned}
$$

This contradicts (3.2), for $0 \leq r_{j}<t_{j}$ and $x_{j} \in \partial B_{\delta}$. This completes the proof.

To prove Theorems 3 5, we need some known results about analytic sets. The reader is referred to [4, pages $14-57$, for the details.

Lemma 7. Let $\mathcal{A}$ be an analytic subset of an open subset of $\mathbb{C}^{n}$. Then for any $p \in \mathcal{A}$, the (complex) dimension $\operatorname{dim}_{p} \mathcal{A}$ of $\mathcal{A}$ at $p$ is at least 1 if and only if $p$ is an accumulated point of $\mathcal{A}$.

Lemma 8. If $\operatorname{dim}_{p} \mathcal{A} \geq 1$, then $\mathcal{A}$ has an irreducible component $\mathcal{B}$ containing $p$, with a pure complex dimension at least 1 .

Lemma 9. Any irreducible component of $\mathcal{A}$ is the closure in $\mathcal{A}$ of some connected component of $\operatorname{reg} \mathcal{A}$, where $\operatorname{reg} \mathcal{A}$ is the set of all regular points of $\mathcal{A}$.

A point $p \in \mathcal{A}$ is called regular, if there is a neighborhood $V_{p}$ of $p$ in $\mathbb{C}^{n}$, such that $V_{p} \cap \mathcal{A}$ is a complex submanifold of $V_{p}$.

Proof of Theorem 3. We first proof the necessity. Assume that there exists an analytic variety $\Sigma$ of pure complex dimension at least 1, consisting of the singularity 0 and periodic orbits of (2.3) of the same period $\tau_{0}$ (note that the period always indicates the least positive period).

By Lemma 6, there exist positive numbers $\delta$ and $T_{0}\left(<\tau_{0}\right)$ such that for each $T \in\left(0, T_{0}\right]$, the system (2.3) has no periodic orbit of period $T$ intersecting $\bar{B}_{\delta}$.

Let $M$ be a prime number such that $\tau_{0} / M<T_{0}$ and let $\Phi(x)=\phi\left(\tau_{0} / M, x\right)$ be the time $\tau_{0} / M$ mapping of the local flow $\phi(t, x)$ of (2.3). Then 0 is the unique fixed point of $\Phi$ located in $\bar{B}_{\delta}$. On the other hand, by the assumption about $\Sigma, 0$ is an accumulated point of $\Sigma$ and all points of $\Sigma$ are fixed points of $\Phi^{M}(x)=\phi\left(\tau_{0}, x\right)$, and then 0 is an accumulated point of fixed points of $\Phi^{M}$.

Thus by Lemma 4, $\Phi^{\prime}(0)$ has an eigenvalue $\Lambda$ such that $\Lambda \neq 1$ but $\Lambda^{M}=1$, which implies by Lemma 5 that $F^{\prime}(0)$ has an eigenvalue $\lambda$ with $\Lambda=e^{\frac{\tau_{0} \lambda}{M}} \neq 1$ and $\Lambda^{M}=e^{\tau_{0} \lambda}=1$. Clearly, $\lambda=\omega i$ fore some real number $\omega \neq 0$. This completes the proof of the necessity.

The sufficiency follows from Theorem 4 directly.

Proof of Theorem 4. Assume $F^{\prime}(0)$ has an eigenvalue $\omega i$ such that $\omega \neq 0$ is a real number and for any other eigenvalue $\lambda$

$$
\lambda /(\omega i) \neq \pm 2, \pm 3, \ldots
$$

Then by Corollary 1 , the origin 0 is an accumulated point of fixed points of the time $\frac{2 \pi}{|\omega|}$ mapping $\Phi=\phi\left(\frac{2 \pi}{|\omega|}, \cdot\right)$ of the local flow $\phi(t, x)$ of (2.3) defined in a neighborhood of the origin.

By Lemma 5, for some ball $B$ centered at the origin $0, \phi(t, x)$ is real analytic on $\left[0, \frac{2 \pi}{|\omega|}\right] \times \bar{B}$ and holomorphic with respect to $x \in \bar{B}$. Let $\mathcal{A}_{B}$ be the set of all fixed points of the mapping $\Phi$ in

$$
V_{B}=\phi\left(\left[0, \frac{2 \pi}{|\omega|}\right] \times B\right)=\left\{\phi(t, x) ; t \in\left[0, \frac{2 \pi}{|\omega|}\right], x \in B\right\} .
$$


Then $\mathcal{A}_{B}$ is an analytic subset of $V_{B}$, and by Lemma 7 we have $\operatorname{dim}_{0} \mathcal{A}_{B} \geq 1$, and then, by Lemma 8 , there exists an irreducible component $\Sigma_{B}$ of $\mathcal{A}_{B}$ containing $p$ with pure complex dimension at least 1.

We first show that $\Sigma_{B} \backslash\{0\}$ consists of periodic orbits of (2.3). It suffices to prove that $\Sigma_{B}$ is invariant by the flow $\phi(t, x)$. Note that by the definition of $V_{B}, \mathcal{A}_{B}$ is invariant by the local flow $\phi$, say, $\phi\left(t, \mathcal{A}_{B}\right)=\mathcal{A}_{B}$ for all real $t$.

By Lemma 9 there exists a connected component $S$ of $\operatorname{Reg} \mathcal{A}_{B}$ such that $\Sigma_{B}$ is the closure of $S$ in $\mathcal{A}_{B}$. Then each $p \in S$ has a neighborhood $U_{p}$ in $V_{B}$, such that $S \cap U_{p}$ is a complex submanifold of $U_{p}$ and $S$ is the only connected component of $\mathcal{A}_{B}$ intersecting $U_{p}$, and then $\Sigma_{B}$ is the only irreducible component of $\mathcal{A}_{B}$ intersecting $U_{p}$ by Lemma 9 . On the other hand, for sufficiently small $\varepsilon>0, \Phi_{\varepsilon}\left(\Sigma_{B}\right)=\phi\left(\varepsilon, \Sigma_{B}\right)$ must intersect $U_{p}$, and then by the fact that $\Phi_{\varepsilon}=\phi(\varepsilon, \cdot)$ is a biholomorphic mapping from $V_{B}$ onto $\Phi_{\varepsilon}\left(V_{B}\right), \Phi_{\varepsilon}\left(\Sigma_{B}\right)$ is also an irreducible component of $\Phi_{\varepsilon}\left(\mathcal{A}_{B}\right)=\mathcal{A}_{B}$. (Recall that $\mathcal{A}_{B}$ is invariant by the flow $\phi(t, x)$.) Therefore, $\Phi_{\varepsilon}\left(\Sigma_{B}\right)$ and $\Sigma_{B}$ are both irreducible components of $\mathcal{A}_{B}$ and both intersect $U_{p}$, and then $\Phi_{\varepsilon}\left(\Sigma_{B}\right)=\Sigma_{B}$, which implies that $\phi\left(t, \Sigma_{B}\right)=\Sigma_{B}$ for all real $t$. Thus $\Sigma_{B}$ is invariant by $\phi$ and then $\Sigma_{B} \backslash\{0\}$ consists of periodic orbits.

It is clear that for each periodic orbit $\mathcal{O} \subset \Sigma_{B} \backslash\{0\}$, the period $T(\mathcal{O})$ of $\mathcal{O}$ equals to $\frac{2 \pi}{|\omega| m_{\mathcal{O}}}$ for some positive integer $m_{\mathcal{O}}$. By Lemma 6, we may assume that $B$ is small enough such that for some fixed $T_{0}>0$, the period of each orbit in $\Sigma_{B} \backslash\{0\}$ is larger than $T_{0}$. Then for each periodic orbit $\mathcal{O} \subset \Sigma_{B}$ we have $T_{0} \leq T(\mathcal{O})=\frac{2 \pi}{|\omega| m_{\mathcal{O}}}$, with

$$
m_{\mathcal{O}} \in\left\{1,2, \ldots, m^{*}\right\},
$$

where $m^{*}$ is the integral part of $2 \pi\left(T_{0}|\omega|\right)^{-1}$.

We assert that if the ball $B$ is small enough, then for each periodic orbit $\mathcal{O} \subset$ $\Sigma_{B} \backslash\{0\}, m_{\mathcal{O}}=1$. Otherwise, by (3.5) there is a fixed integer $m>1$, and a sequence $\left\{x_{k}\right\} \subset \Sigma_{B} \backslash\{0\}$, such that $x_{k} \rightarrow 0$ as $k \rightarrow \infty$ and the periodic orbit passing through $x_{k}$ has period $\frac{2 \pi}{|\omega| m}$ for each $k$, say, $\phi\left(\frac{2 \pi}{|\omega| m}, x_{k}\right)=x_{k}$ for each $k$.

Let $M$ be any given prime number with $\frac{2 \pi}{|\omega| m M}<T_{0}$. Then 0 is an isolated fixed point of $\Theta(x)=\phi\left(\frac{2 \pi}{|\omega| m M}, x\right)$, but 0 is an accumulated point of fixed points of $\Theta^{M}$, for

$$
\Theta^{M}\left(x_{k}\right)=\phi\left(\frac{2 \pi}{|\omega| m}, x_{k}\right)=x_{k}, k=1,2, \ldots
$$

Therefore, by Lemma 4, the Jacobian matrix $\Theta^{\prime}(0)$ must have an eigenvalue $\Lambda$ with $\Lambda \neq 1$ but $\Lambda^{M}=1$. Hence, by (1.3), we have $\Lambda=e^{\frac{2 \pi}{\left.\right|^{2 m M} \lambda}}$ for some eigenvalue $\lambda$ of $F^{\prime}(0)$ such that $\lambda \neq 0$ and $\frac{2 \pi}{|\omega| m} \lambda$ is a multiple of $2 \pi i$, and then $\lambda /(\omega i)= \pm k m$ for some fixed positive integer $k$, which contradicts (3.4). Therefore, we have $m=1$. The proof is complete.

Proof of Theorem 5. Assume that $\lambda_{1}, \ldots, \lambda_{n}$ are the $n$ eigenvalues of the Jacobian matrix $F^{\prime}(0)$ of $F$ at 0 , with $\lambda_{1}=\omega i$ and

$$
\lambda_{l} / \lambda_{1} \neq 0, \pm 1, \pm 2, \pm 3, \ldots, l=2,3, \ldots, n .
$$

Ignoring a linear transform of the phase space, we may assume that the Jacobian matrix $F^{\prime}(0)$ is lower triangular and has $\lambda_{1}, \lambda_{2}, \ldots, \lambda_{n}$ down its main diagonal. We write this by

$$
F^{\prime}(0)=\left(\lambda_{1}, \lambda_{2}, \lambda_{3}, \ldots, \lambda_{n}\right)
$$


By Lemma [5] there exists a ball $B$ centered at the origin 0 such that the local flow $\phi(t, x)$ of $(2.3)$ is real analytic on $\left[0, \frac{2 \pi}{|\omega|}\right] \times \bar{B}$ and complex holomorphic with respect to $x \in B$.

By (3.7) and Lemma 5.

$$
\Lambda_{l}=e^{\frac{2 \pi}{|\omega|} \lambda_{l}}, l=1,2, \ldots, n,
$$

are all the eigenvalues of the Jacobian matrix $\Phi^{\prime}(0)$ of the time $\frac{2 \pi}{|\omega|}$ mapping $\Phi(x)=$ $\phi\left(\frac{2 \pi}{|\omega|}, x\right)$ at 0 , and by (3.6) we have

$$
\Lambda_{l} \neq 1, l=2, \ldots, n .
$$

It also follows from (3.7) and Lemma 5 that $\Phi^{\prime}(0)=\left(1, \Lambda_{2}, \Lambda_{3} \ldots, \Lambda_{n}\right)$ is a lower triangular matrix which has $1, \Lambda_{2}, \Lambda_{3} \ldots, \Lambda_{n}$ down its main diagonal. Therefore, putting $x=\left(x_{1}, x_{2}, \ldots, x_{n}\right)$ and $\Phi=\left(\varphi_{1}, \varphi_{2}, \ldots, \varphi_{n}\right)$, by (3.8) we have

$$
\left.\operatorname{det}\left(\frac{\partial\left(\varphi_{2}, \varphi_{3}, \ldots, \varphi_{n}\right)}{\partial\left(x_{2}, x_{3}, \ldots, x_{n}\right)}-I_{n-1}\right)\right|_{x=0} \neq 0
$$

where $I_{n-1}$ is the $(n-1) \times(n-1)$ unit matrix. So, by the implicit function theorem, there uniquely exist one variable complex holomorphic function $x_{l}=x_{l}\left(x_{1}\right), l=$ $2, \ldots, n$, defined in a neighborhood of the origin in the complex plane $\mathbb{C}$, solving the system of the equations

$$
\varphi_{l}\left(x_{1}, x_{2}, \ldots, x_{n}\right)=x_{l}, l=2, \ldots, n,
$$

in a neighborhood of the origin 0 , with

$$
x_{l}(0)=0, l=2,3, \ldots, n .
$$

Therefore, in a neighborhood of the origin 0 in $\mathbb{C}^{n}$, the fixed point equation

$$
\Phi\left(x_{1}, x_{2}, \ldots, x_{n}\right)=\left(x_{1}, x_{2}, \ldots, x_{n}\right)
$$

is equivalent to the system of the $n$ equations

$$
\left\{\begin{array}{l}
x_{1}=\varphi_{1}\left(x_{1}, x_{2}\left(x_{1}\right), \ldots, x_{n}\left(x_{1}\right)\right), \\
x_{l}=x_{l}\left(x_{1}\right), l=2, \ldots, n .
\end{array}\right.
$$

By Corollary [1, 0 is an accumulated point of fixed points of $\Phi$, and then 0 is an accumulated point of zeros of the holomorphic function $x_{1}-\varphi_{1}\left(x_{1}, x_{2}\left(x_{1}\right), \ldots, x_{n}\left(x_{1}\right)\right)$ which is defined in a neighborhood of the origin 0 in $\mathbb{C}$. Therefore, we have

$$
\varphi_{1}\left(x_{1}, x_{2}\left(x_{1}\right), \ldots, x_{n}\left(x_{1}\right)\right) \equiv x_{1}
$$

in a neighborhood of $x_{1}=0$ in $\mathbb{C}$, and then (3.11) reads

$$
\left\{\begin{array}{l}
x_{1}=x_{1} \\
x_{l}=x_{l}\left(x_{1}\right), l=2, \ldots, n .
\end{array}\right.
$$

It is clear that there exists a positive number $\delta$ such that

$$
\Sigma_{\delta}^{*}=\left\{\left(x_{1}, \varphi_{2}\left(x_{1}\right), \ldots, \varphi_{n}\left(x_{1}\right)\right) ; x_{1} \in \mathbb{C},\left|x_{1}\right|<\delta\right\}
$$

is an analytic disk in $\Delta^{n}$, which contains 0 by (3.9).

By the equivalence of (3.10) and (3.12), in a neighborhood of $0, \Sigma_{\delta}^{*}$ coincides with the set $\mathcal{A}_{B}$ of all fixed points of $\Phi$ in

$$
V_{B}=\phi\left(\left[0, \frac{2 \pi}{|\omega|}\right] \times B\right),
$$


and then $0 \in \mathcal{A}_{B}$ has a neighborhood $U_{0}$ in $\mathcal{A}_{B}$ so that $U_{0}$ is an analytic disk in $\mathbb{C}^{n}$. On the other hand, by Theorem 4 there exists an analytic variety $\Sigma$ of pure complex dimension at least 1, consisting of 0 and periodic orbits of the same period $\frac{2 \pi}{\omega}$ of the system (2.3), and then by the equivalence of (3.10) and (3.12), $0 \in \Sigma$ has a neighborhood $V_{0}$ in $\Sigma$ such that $V_{0} \subset U_{0}$, which implies that the dimension of $\Sigma$ is equal to 1 , and then $V_{0}$ coincides with $U_{0}$ in a neighborhood of 0 .

Now, we can conclude that $\Sigma, \Sigma_{\delta}^{*}$ and $\mathcal{A}_{B}$ coincide in a neighborhood of the origin. Therefore, when $B$ is small enough, $\mathcal{A}_{B}$ is contained in $\Sigma \cap \Sigma_{\delta}^{*}$, and then $\mathcal{A}_{B}$ contains an analytic disk $D$ consisting of 0 and periodic orbits of the same period $\frac{2 \pi}{|\omega|}$.

The above argument also shows that any analytic disk $D$ consisting of 0 and periodic orbits of (2.3) with the same period $\frac{2 \pi}{|\omega|}$ coincides with $\Sigma_{\delta}^{*}$ in a neighborhood of 0 , which implies the uniqueness, and the proof is complete.

\section{Appendix}

Proof of Proposition 1. By the given condition, there exists a ball $B \subset \Delta^{n}$ with center 0 such that both $f$ and $f^{m}$ are well defined in a neighborhood of $\bar{B}$ and 0 is the unique fixed point of both $f$ and $f^{m}$ in $\bar{B}$.

Let $\lambda$ be an eigenvalue of $f^{\prime}(0)$ that is a primitive $m$-th root of 1 . Then the Jacobian matrix $\left(f^{m}\right)^{\prime}(0)=\left(f^{\prime}(0)\right)^{m}$ of $f^{m}$ at 0 has the eigenvalue $\lambda^{m}=1$, and then by Lemma 1 we have

$$
\mu_{f^{m}}(0) \geq 2 .
$$

We first assume that none of eigenvalues of $f^{\prime}(0)$ equals 1 . Then applying Lemma 1 once more we have

$$
\mu_{f}(0)=1
$$

We can construct a sequence $f_{k}: \Delta^{n} \rightarrow \mathbb{C}^{n}$ of holomorphic mappings, converging to $f$ uniformly, such that for each $k, 0$ is a simple fixed point of both $f_{k}$ and $f_{k}^{m}$, say, $f_{k}(0)=f_{k}^{m}(0)=0$, but neither $f_{k}^{\prime}(0)$ nor $\left(f_{k}^{m}\right)^{\prime}(0)$ has eigenvalue 1 . This can be accomplished by small perturbations of the linear part of $f$ at 0 .

It is clear that for sufficiently large $k, f_{k}^{m}$ is well defined on $\bar{B}$ and $f_{k}^{m}$ converges to $f^{m}$ uniformly on $\bar{B}$ as $k$ tends to $\infty$. Therefore, by (4.1), Lemma 3 and the condition that 0 is a simple fixed point of $f_{k}^{m}$, we conclude that for sufficiently large $k, f_{k}^{m}$ has another fixed point $x_{k} \neq 0$ in $B$. On the other hand, since 0 is the unique fixed point of $f$ in $\bar{B}$, by Lemma 3 and (4.2), for sufficiently large $k, 0$ is the unique fixed point of $f_{k}$ in $B$. Thus, we have $f_{k}\left(x_{k}\right) \neq x_{k}$.

Now that $f_{k}\left(x_{k}\right) \neq x_{k}, f_{k}^{m}\left(x_{k}\right)=x_{k}$ and $m$ is prime, $x_{k}$ is a periodic point of $f_{k}$ with period $m$, say, the periodic orbit $\Gamma\left(x_{k}\right)=\left\{x_{k}, f_{k}\left(x_{k}\right), \ldots, f_{k}^{m-1}\left(x_{k}\right)\right\}$ contains $m$ distinct points. Since 0 is the unique fixed point of $f^{m}$ in $\bar{B}$, by the convergence of $f_{k}^{m}$ we have that $x_{k} \rightarrow 0$ as $k \rightarrow \infty$, and then by the condition $f(0)=0$ and the convergence of $f_{k}$, the periodic orbit $\Gamma\left(x_{k}\right)$ is contained in $B$ for sufficiently large $k$. Thus for sufficiently large $k, 0, x_{k}, f_{k}\left(x_{k}\right), \ldots, f_{k}^{m-1}\left(x_{k}\right)$ are $m+1$ distinct fixed points of $f_{k}$ in $B$. By Lemma 3 , we then have

$$
\mu_{f^{m}}(0) \geq m+1 \text {. }
$$

Therefore, (1.1) holds. 
In general, we can consider the mapping

$$
f_{\varepsilon}=\left(\varepsilon_{1} z_{1}, \ldots, \varepsilon_{n} z_{n}\right)+f\left(z_{1}, \ldots, z_{n}\right)
$$

where $\varepsilon=\left(\varepsilon_{1}, \ldots, \varepsilon_{n}\right)$ is so chosen that $f_{\varepsilon}^{\prime}(0)$ has eigenvalue $\lambda$ but none other eigenvalue equals 1 . It is easy to see that we can choose $\varepsilon$ sufficiently small so that $f_{\varepsilon}^{m}$ is sufficiently close to $f^{m}$, and then by Lemma 3, we have $\mu_{f^{m}}(0) \geq \mu_{f_{\varepsilon}^{m}}(0) \geq m+1$. This completes the proof.

\section{REFERENCES}

[1] Brickman, L. \& Thomas, E. S., Conformal equivalence of analytic flows, J. Differential Equations 25 (1977), no. 3, 310-324. MR0447674 (56:5984)

[2] Chavarriga, J. \& Sabatini, M., A Survey of isochronous centers, Qualitative theory of dynamical systems, 1 (1999), 1-70. MR1747197 (2001c:34056)

[3] Cherkas, L. A., Romanovskii, V. G. \& Zołądek, H., The centre conditions for a certain cubic system, Planar nonlinear dynamical systems (Delft, 1995), Differential Equations Dynam. Systems 5 (1997), no. 3-4, 299-302. MR1660202 (99i:34041)

[4] Chirka, E. M., Complex analytic sets, Translated from the Russian by R. A. M. Hoksbergen, Mathematics and its Applications (Soviet Series), 46. Kluwer Academic Publishers Group, Dordrecht (1989). MR.1111477 (92b:32016)

[5] Christopher, C. J. \& Devlin, J., Isochronous centers in planar polynomial systems, SIAM J. Math. Anal. 28 (1997), no. 1, 162-177. MR1427732 (97k:34058)

[6] Gregor, J., Dynamical systems with regular hand-side, Pokroky Mat. Fys. Astronom. 3 (1958), 153-160 (Zbl 081.30802).

[7] Hajek, O., Notes on meromorphic dynamical systems, I-III, Czechoslovak Math. J. 16 (1966), 14-40. MR0194661 (33:2870a) MR0194662 (33:2870b) MR0194663 (33:2870c)

[8] Lukaševič, N.A., The isochronism of a center of certain systems of differential equations, Differ. Uravn. 1 (1965), 295-302. MR0197863 (33:6023)

[9] Milnor, J., Singular Point of Complex Hypersurfaces, Ann. Math. Study 61. Princeton University Press, New Jersey (1968). MR.0239612 (39:969)

[10] Needham, D. J., A centre theorem for two-dimensional complex holomorphic systems and its generalization., Proc. Roy. Soc. London Ser. A 450 (1995), no. 1939, 225-232. MR1349506 $(96 \mathrm{j}: 34080)$

[11] Needham, D. J. \& McAllister, S., Centre families in two-dimensional complex holomorphic dynamical systems, R. Soc. Lond. Proc. Ser. A Math. Phys. Eng. Sci. 454 (1998), no. 1976, 2267-2278. MR1639872 (99d:34010)

[12] Paluszny, M., On periodic solutions of polynomial ODEs in the plane, J. Differential Equations 53 (1984), no. 1, 24-29. MR0747404 (86g:34054)

[13] Sabatini, M., Dynamics of commuting systems on two-dimensional manifolds, Ann. Mat. Pura Appl. (4) 173 (1997), 213-232. MR1625543 (99f:34071)

[14] Shub, M., \& Sullivan, D., A remark on the Lefschetz fixed point formula for differentiable maps, Topology 13 (1974), 189-191. MR0350782 (50:3274)

[15] Villarini, M., Regularity properties of the period function near a center of a planar vector field, Nonlinear Anal. 19 (1992), no. 8, 787-803. MR1186791 (93j:34061)

[16] Zhang, G. Y., Multiplicities of fixed points of holomorphic maps in several complex variables, Sci. China Ser. A 44 (2001), no. 3, 330-337. MR1828756 (2002b:37026)

[17] Zhang, G. Y., Bifurcations of periodic points of holomorphic maps from $\mathbb{C}^{2}$ into $\mathbb{C}^{2}$. Proc. London Math. Soc., 79(3) (1999), 353-380. MR1702246 (2000f:32027)

Department of Mathematical Sciences, Tsinghua University, Beijing 100084, People's Republic of China

E-mail address: gyzhang@math.tsinghua.edu.cn

E-mail address: gyzhang@mail.tsinghua.edu.cn 